\title{
Novel Therapeutic Interventions in Treating Patients with COVID-19 Pneumonia, COVID-19 Acute Respiratory Syndrome and Severe COVID-19 Illness and Promising Vaccine Candidates
}

\author{
Attapon Cheepsattayakorn ${ }^{1 *}$ and Ruangrong Cheepsattayakorn ${ }^{2}$ \\ ${ }^{1} 10^{\text {th }}$ Zonal Tuberculosis and Chest Disease Center, Chiang Mai, Thailand \\ ${ }^{2}$ Department of Pathology, Faculty of Medicine, Chiang Mai University, Chiang \\ Mai, Thailand \\ *Corresponding Author: Attapon Cheepsattayakorn, 10th Zonal Tuberculosis and \\ Chest Disease Center, Chiang Mai, Thailand.
}

Received: March 23, 2020

Published: April 17, 2020

(C) All rights are reserved by Attapon

Cheepsattayakorn., et al.
Patients with COVID-19 pneumonia were detected in Wuhan city, China since late December 2019. More and more cases have been identified in other areas outside Wuhan city of China and abroad, particularly in Italy, Iran and other European countries, including the United Kingdom and the United States. A total of 77,779 confirmed COVID-19-infected cases were in China, as of February 24, 2020. As of Friday morning (March 20, 2020), at least 246,275 COVID-19-infected cases have been diagnosed worldwide and 14,250 of which are in the United States. At least 10,038 deaths has been accounted, globally, including 205 deaths in the United States. Currently, there is no effective treatment for COVID-19-infected patients. Some recent clinical studies demonstrated that alveolar epithelial cells and capillary endothelial cells of the patients' lungs were damaged contributing to acute lung injury. Inhibition of pulmonary inflammatory response is hypothesized to be the key to cure the patients with COVID-19 pneumonia. Chloroquine, a potential broad-spectrum antiviral agent found in 2006, can interfere with the virus's ability to replicate in two ways. First, the rug enters compartments called "endosomes" within the cell membrane. Endosomes tend to be slightly acidic, the chemical structure of the drug boosts their $\mathrm{pH}$, making the compartments more basic. Many viruses acidify endosomes in order to breach the cell membrane, release their genetic material and initiate replication. Chloroquine blocks this critical step. Second, chloroquine also prevents SARS$\mathrm{CoV}$ from plugging into a receptor called "angiotensin-converting enzyme 2 (ACE 2)" on primate cells, demonstrated in the 2005 report. The virus sets off a chemical process that alters the structure of the ACE 2 receptor and allows the virus to infect when the virus inserts its spike protein into the ACE 2 receptor. Chloroquine blocks COVID-19 infection at low-micromolar concentration, with a half-maximal effective concentration (EC50) of $1.13 \mu \mathrm{M}$ and a half-cytotoxic concentration (CC50) greater than $100 \mu \mathrm{M}$. An appropriate dose of chloroquine is to be undermined in this process. The investigators expect that whatever pertained to SARS-CoV-1 might apply to SARS-CoV-2 (COVID-19).
In February 2020, Manli Wang, a virologist in a research group of the Chinese Academy of Sciences demonstrated that chloroquine successfully inhibited the spread of SARS-CoV-2 (COVID-19) in cultured human cells. Preliminary reports from China, South Korea, and France indicated that the treatment was effective in COVID-19-infected patients. Some hospitals in the United States have initiated administering chloroquine in treating severely COVID-19-infected patients. Additionally, the United States Food and Drug Administration (US FDA) is organizing a large clinical trial to formally evaluate the chloroquine's effects. The US FDA will take all steps to ensure chloroquine remains available for patients who take it to treat severe and life-threatening illnesses, such as lupus. Nevertheless, due to the overdose of chloroquine can contribute to acute poisoning or death in humans and a short supply of chloroquine in China, Wang's research team also studied the closely related drug "hydroxychloroquine" that shares a similar chemical structure and reveals lower toxicity in animals than chloroquine and also remains widely available in treating lupus and rheumatoid arthritis. Wang and colleagues reported in the journal "Cell Discovery" published on March 18, 2020 that hydroxychloroquine can prevent SARS-CoV-2 (COVID-19) replication. Seven clinical trials had been registered in the Chinese Clinical Trial Registry to test hydroxychloroquine's effectiveness against COVID-19 infection. The University of Minnesota, United States is studying whether taking hydroxychloroquine can protect persons living with COVID-19-infected patients from catching the virus themselves. According to the American Society of Health-System Pharmacists, both chloroquine and hydroxychloroquine have been in short supply since earlier March 2020. Nevertheless, on March 19, 2020, a drug company from Germany donated 3 million tablets of chloroquine phosphate to the US federal government.

In a single protocol was studied from early March 2020 to March 16, 2020, Thirty-six French COVID-19-confirmed patients were included to receive $600 \mathrm{mg}$ of hydroxychloroquine daily. Azithromy-

Citation: Attapon Cheepsattayakorn and Ruangrong Cheepsattayakorn. "Novel Therapeutic Interventions in Treating Patients with COVID-19 Pneumonia, COVID-19 Acute Respiratory Syndrome and Severe COVID-19 Illness and Promising Vaccine Candidates". Acta Scientific Microbiology 3.5 (2020): $97-99$. 
cin was added to the treatment protocol depending on their clinical presentation. Nasopharyngeal swabs for testing their viral load were performed daily in a hospital setting. Absence and presence of COVID-19 ay Day6-post inclusion was considered the end point. Eight patients presented with lower respiratory tract symptoms, 22 cases presented with upper respiratory tract infection, whereas 6 cases were asymptomatic. Twenty cases were treated and demonstrated a significant decrease of the viral load at Day6-post inclusion, whereas adding azithromycin to hydroxychloroquine was significantly more efficient in viral load reduction.

Remdesivir, a nucleoside analogue with a broad-spectrum antiviral activity and as being in the US clinical trials with near approval for use by the US FDA has been also studied in France by Gautret., et al. from Marseille University. The investigators feel optimistic about the French research data. Previous studies conducted by the researchers from the University of Alberta, Canada and Gilead involving cell cultures and animal models has demonstrated that remdesivir can block the replication of a variety of coronaviruses, hypothesized by blocking the RNA-dependent RNA polymerase, a particular enzyme that is required for viral replication. Remdesivir potently blocks COVID-19 infection at low-micromolar concentration and has a high selectivity index (half-maximal effective concentration (EC50), $0.77 \mu \mathrm{M}$; half-cytotoxic concentration (CC50) > $100 \mu \mathrm{M}$; SI > 129.87). A previous study in the US reported that remdesivir treatment demonstrated promising results. For evaluating the efficacy and safety of remdesivir in patients with COVID-19 disease, a randomized placebo-controlled, double-blind, multicentric phase III clinical trials was initiated on February 5, 2020 in China. The subjects in the study group received an initial dose of $200 \mathrm{mg}$ of remdesivir and a subsequent dose of $100 \mathrm{mg}$ for 9 consecutive days through intravenous infusion in addition to routine treatment. The control group received routine treatment and the same dose of a placebo. By the end of April 2020, the clinical trial is expected to be concluded. Remdesivir was developed by the US pharma giant Gilead Sciences Inc. and previously was tried to treated Middle-East-Respiratory Syndrome (MERS) and Ebola. The Credit Suisse pharma team declares that remdesivir is the most advanced novel drug in treating patients with COVID-19 disease but concerning about the supply.

On February 4, 2020, investigators in China announced that darunavir inhibited COVID-19 viral replication at a concentration of $300 \mu \mathrm{M}$ in vitro and its inhibition efficiency was 280 -fold that in the control group. Type II transmembrane serine protease (TMSPSS2) inhibitors and BCR-ABL kinase inhibitor-imatinib are other potential drugs. TMSPSS2 inhibitors would block the entry of the cellular protease, TMPRSS2 into the target cells via ACE 2 receptor. Imatinib inhibits the fusion of virions with the endosomal membrane (anti-coronal activity). On January 25, 2020, 30 drugs with potential antiviral activity against COVID-19 performed through the drug screening in silicon and an enzyme activity test cinanserin, cyclosporin A, TDZD-8, PX-12, tideglusib, ebselen, shikonin, carmofur, disulfiram, chalcone, polydatin, deoxyrhapontin, montelukast, raltegravir, maribavir, elvitegravir, bortezomib, abacavir, presatovir, enzaplatovir, fosamprenavir, tipranavir, darunavir, atazanavir, remdesivir, ritonavir, carfilzomib, lopinavir, saquinavir, and indinavir were reported by a joint research team of the Shanghai Institute of Materia Medica and Shanghai Tech University. The same research also demonstrated that Chinese herbal medicines, such as Radix Sophorae Tonkinensis and Rhizoma Polygoni Cuspidati may contain ingredients against COVID-19. Until now, it has been difficult to get the polymerase complex that contains multiple proteins to function in a test tube.

A drug company in the US has announced the initiation of a randomized controlled clinical trial of sarilumab, an antibody to the interleukin (IL)-6 receptor, to evaluate whether the modification of the lung inflammatory response by therapeutic intervention provides the benefit to COVID-19-infected patients. The latest edition (the 6th edition) China national Guidelines for COVID-19 treatment, issued on February 18, 2020, recommends interferon- $\alpha$ (IFN- $\alpha$ ), lopinavir/ritonavir, ribavirin, chloroquine phosphate and arbidol for treatment agents. IFN- $\alpha$ is administered via vapor inhalation at a dose of 5 million Units (and $2 \mathrm{ml}$ of sterile water for infection) for adults, 2 times daily. Lopinavir/ritonavir is administered orally at a dose of $400 \mathrm{mg} / 100 \mathrm{mg}$ for adults, 2 times daily. Ribavirin is administered via intravenous infusion at a dose of $500 \mathrm{mg}$ each time for adults, 2 to 3 times daily in combination with IFN- $\alpha$ or lopinavir/ritonavir. Chloroquine phosphate is administered orally at a dose of $500 \mathrm{mg}$ (300 mg for chloroquine) each time for adults, 2 times daily. Arbidol is administered orally at a dose of $200 \mathrm{mg}$, each time, 3 times daily. Arbidol effectively inhibits COVID-19 infection at a concentration of $10-30 \mu \mathrm{M}$ in vitro. The duration of all treatment options is no more than 10 days.

On February 15, 2020, favipiravir, a new type of RNA-dependent RNA polymerase (RdRp) inhibitor that was first approved in Japan in March 2014 for establishing preparedness against the possible outbreak of novel or re-emerging influenza virus infections was approved for treatment of COVID-19 disease in China. Currently, favipiravir is undergoing clinical trials in treating COVID-19 disease. In addition to favipiravir's anti-influenza virus activity, it can block the replication of alpha-, arena-, bunya-, filo-, flavi-, and other

Citation: Attapon Cheepsattayakorn and Ruangrong Cheepsattayakorn. "Novel Therapeutic Interventions in Treating Patients with COVID-19 Pneumonia, COVID-19 Acute Respiratory Syndrome and Severe COVID-19 Illness and Promising Vaccine Candidates". Acta Scientific Microbiology 3.5 (2020): 97-99. 
RNA viruses. Favipiravir inhibits RNA polymerase activity by the conversion of favipiravir into an active phosphoribosylated form (favipiravir-RTP) in cells and is recognized as a substrate by viral RNA polymerase. A clinical trial on favipiravir for the treatment of COVID-19 disease was initiated by the Third People's Hospital of Shenzhen and the Clinical Medical Research Center of the National Infectious Diseases, China on February 14, 2020 achieved the promising results. The preliminary results from a total of 80 patients with COVID-19 disease, including the control group and the experimental group demonstrated that favipiravir had more potent antiviral activity than that of lopinavir/ritonavir. Favipiravir treatment group demonstrated no significant adverse reactions and had significantly fewer adverse effects than the lopinavir/ritonavir group.

"Convalescent plasma" therapy has been working on some time by the US FDA. This is not a proven treatment, but is a possible treatment. The immunoglobulins in the previously COVID-19-exposed individuals' circulating virus-free convalescent plasma could potentially provide a benefit to severely COVID-19-infected patients. Currently, several trials on mesenchymal stem cells in treating patients with COVID-19 pneumonia are ongoing. For examples, trial sponsored by Innovative Precision Medicine Group (IPM), China, Wuhan Houshenshan Hospital, Wuhan, China, Tianjin Haihe Hospital, VCANBIO CELL and GENE ENGINEERING CORP., LTD., China, Shenzhen Third People' s Hospital, and Fifth Affiliated Hospital, Sun Yat-Sen University, China (NCT04252118); trial on human umbilical cord mesenchymal stem cell treatment for COVID-19-pneumonia patients sponsored by Wuhan Union Hospital, China and Wuhan Hamilton Bio-technology Co., Ltd., China (NCT04273646); trial on inhalation of mesenchymal stem cell exosomes in treating COVID-19-pneumonia patients sponsored by Ruijin Hospital, China, Shanghai Public Health Clinical Center, Wuhan Jinyintan Hospital, Wuhan, China, and Cellular Biomedicine Group Ltd. (NCT04276987); trial on human umbilical cord mesenchymal stem cells for treatment of COVID-19-pneumonia patients sponsored by Puren Hospital Affiliated to Wuhan University of Science and Technology and Wuhan Hamilton Bio-technology Co., Ltd. (NCT04293692); trial on dental pulp mesenchymal stem cell treatment for COVID-19-pneumonia patients sponsored by CART (Shanghai) Biotechnology Co., Ltd. (NCT04302519); and trial on mesenchymal stem cells in treating patients with COVID-19 pneumonia sponsored by Beijing 302 Hospital, VCANBIO CELL and GENE ENGINEERING CORP.,LTD., China, Wuhan Huoshenshan Hospital, Wuhan, China, Tianjin Haihe Hospital, Shenzhen Third People's Hospital, Fifth Affiliated Hospital, Sun Yat-Sen University, China, Wuhan Union Hospital, China, and West China Hospital (NCT04288102). Trials on several vaccine candidates, currently are also ongoing, for examples; phase I trial sponsored by Moderna Therapeutics, CanSino Biologics, Arcturus Therapeutics (Preclinical stage), BioNTech (Preclinical stage), CureVac (Preclinical stage), GlaxoSmithKline (Preclinical stage), Inovio Pharmaceuticals (Preclinical stage), Johnson and Johnson (Preclinical stage), and Pfizer (Preclinical stage), Sanofi (Preclinical stage). Usually, vaccine development takes more than 5 years and requires much capital investment. There is no guarantee of success though the traditional pharma giants' experience involving seasonal flu, particularly their specializing in mRNA molecules that are used to instruct the human body to produce its own response to combat a range of diseases.

In conclusion, currently, there are no finally verified antivirals and vaccine candidates specific to COVID-19. Further preclinical and clinical trials are urgently needed to successfully treat patients with COVID-19 disease and preventing individuals from COVID-19 infection.

\section{Assets from publication with us}

- Prompt Acknowledgement after receiving the article

- Thorough Double blinded peer review

- Rapid Publication

- Issue of Publication Certificate

- High visibility of your Published work

Website: https://www.actascientific.com/

Submit Article: https://www.actascientific.com/submission.php Email us: editor@actascientific.com

Contact us: +919182824667

Citation: Attapon Cheepsattayakorn and Ruangrong Cheepsattayakorn. "Novel Therapeutic Interventions in Treating Patients with COVID-19 Pneumonia, COVID-19 Acute Respiratory Syndrome and Severe COVID-19 Illness and Promising Vaccine Candidates". Acta Scientific Microbiology 3.5 (2020): $97-99$. 